\title{
Scanning X-Ray Microscopy imaging of strain relaxation and fluctuations in thin patterned SiGe-on-insulator nanostructures
}

G. Girard, ${ }^{1}$ R. Berthelon, ${ }^{2,3,4}$ F. Andrieu, ${ }^{3}$ S. J. Leake, ${ }^{1}$ G. A. Chahine, ${ }^{5}$ T. Schülli, ${ }^{1}$ J. Eymery, ${ }^{6}$ and $\mathrm{V}$. Favre-Nicolin ${ }^{1,7, a)}$

1) ESRF-The European Synchrotron, 38043 Grenoble Cedex 9, France

${ }^{2)}$ STMicroelectronics, 850 rue Monnet, B.P. 16, F-38926 Crolles, France

3) Univ. Grenoble Alpes, CEA, LETI, 17 rue des Martyrs, 38000 Grenoble, France

4) CEMES-CNRS, 29 Rue Jeanne Marvig, 31055 Toulouse Cedex 4, France

5) Univ. Grenoble Alpes, CNRS, Grenoble INP, SIMAP, 38000 Grenoble, France

6) Univ. Grenoble Alpes, CEA, IRIG, MEM, NRS, 17 rue des Martyrs, 38000 Grenoble, France

7) Univ. Grenoble Alpes, 38000 Grenoble, France

(Dated: January 24, 2021)

Strain engineered performance enhancement in SiGe channels for p-MOSFETs is one of the main drivers for the development of microelectronics technologies. Thus, there is a need for precise and accurate strain mapping techniques with small beams. Scanning X-Ray Diffraction Microscopy (SXDM) is a versatile tool that allows measuring quantitative strain maps on islands as thin as $13 \mathrm{~nm}$ quickly. From the high velocity and robustness of the technique, statistical information can be extracted for a large number of individual islands of different sizes. In this letter, we used the advantages of SXDM to demonstrate the effectiveness of the condensation method used to grow ultra-thin layers of strained SiGe and to determine their relaxation lengths at patterned interfaces.

\footnotetext{
a) Electronic mail: favre@esrf.fr
} 


\section{INTRODUCTION}

Electronic properties of semiconductor nano-structures are strongly influenced by their strain state, and many examples have already shown that strain engineering was an efficient tool to boost the microelectronics technologies. ${ }^{1-3}$ For example, the introduction of strain in the channel of highly efficient transistors (Metal Oxide Semiconductor Field-Effect Transistors, MOSFETs) since the $65 \mathrm{~nm}$ technology node allowed the pursuit of Moore's law and enhancing performance in logic gates. ${ }^{4}$

The latest developments have led to a high-performance 14 nm-technology using Fully Depleted Silicon On Insulator (FDSOI) transistors and the integration of a strained SiGe channel in p-type MOSFETs. ${ }^{5-7}$ The choice of strained SiGe as a channel was driven by two factors : firstly, hole mobility in Germanium is higher than in Silicon and secondly, heteroepitaxy of SiGe on Si yields a biaxial compressive stress in the layer, which also enhances the hole mobility of a SiGe p-MOS channel. ${ }^{8}$ In general, the mechanical loading of the transistor channel can be performed by four basic methods by using a: (i) ContactEtch Stop Layer (CESL), e.g. an amorphous layer typically made of Silicon nitride to precisely control a chemical etching (and elastic relaxation) process, (ii) Stress Memorization Technique (SMT), depositing a high tensile stressed silicon nitride (called $\mathrm{SiN}_{x}$ hereafter) on top of the source and drain, the whole structure is then recrystallized and finally, the $\mathrm{SiN}_{x}$ removed, (iii) Pseudomorphic Epitaxy of the source and drain in a material with different lattice parameter than the Silicon substrate, and ultimately (iv) Substrate Engineering which aims to use a modified substrate by epitaxy or bonding transfer of a set of non-matching materials, such as the SiGe-on-insulator (SGOI) studied in this letter. ${ }^{9}$

For such devices, the characterization and understanding of the strain evolution along the transistors integration flow for different design layout/geometries is key to guarantee the predictability of the electrical models and to finally achieve the maximum performance of the circuits. As the size of functional nanostructures is reduced, the ability to map strain at the nanoscale has become essential.

While Transmission Electron Microscopy allows measuring high spatial-resolution strain maps on single objects, it involves destroying the sample and changing the strain state with sample preparation. Also due to sample thinning, it cannot analyze a large number of nano-structures. Raman spectroscopy is based on the knowledge of the phonon mode 
dependence on composition and strain tensor ${ }^{10}$ and a precise determination of phonon shift coefficients $^{11,12}$. This method has been extensively used for the measurement of SiGe nanostructures, e.g. stripes and patterns ${ }^{13,14}$. This technique is also limited by both; the low excitation probability of the Raman process leading to heating effects, and by its resolution related to the optical limit ${ }^{15}$.

$\mathrm{X}$-ray diffraction is also a choice method for strain determination in nano-structures: it is for example possible to determine the average strain field in an assembly of identical nano-objects, using phase retrieval techniques ${ }^{16,17}$. Using modern X-ray focusing optics, micro-diffraction has been increasingly used for strain determination in individual heterostructures $^{18,19}$. Recently, an optimized Scanning X-Ray Diffraction Microscopy (SXDM) technique has been developed at the ESRF ID01 beamline ${ }^{20,21}$ which enables the quantitative determination of strain in nanostructures down to about $100 \mathrm{~nm}$ with a strain sensitivity below $10^{-4}$.

SXDM takes advantage of the Bragg diffraction condition to yield information with different resolution levels: the beam size defines the real-space resolution of the scanning probe, while the beam divergence defines the reciprocal space resolution, and thus the strain resolution. Note that while X-ray Bragg Ptychography ${ }^{22-24}$ can be used to yield a higher resolution strain map, it requires isolating the coherent X-ray scattering from the studied nano-structure, which is not always possible due to the proximity of the substrate diffraction peak.

\section{SAMPLES}

While SOI is anticipated to become a major substrate for future electronics, most of the expected improvements will depend on the CMOS process and in particular on the epitaxy of defect-free ultra-thin Si and SiGe layers. Up to now, two main techniques which will be illustrated in this paper, have become more interesting to produce thin SiGe layers with high crystalline quality. The first approach consists of growing an epitaxial relaxed SiGe layer on $\mathrm{Si}$ (involving for example composition and strain gradients) and uses a combination of implantation and wafer bonding processes to transfer this SiGe layer to an SOI substrate. ${ }^{25-27}$

The second technique to produce SGOI involves the so-called Germanium condensation process (see Figure S1 in the supplementary materials for schematic of the condensation 
process and a sample description). ${ }^{28-32}$ The initial substrate results from the epitaxial growth by Reduced Pressure-Chemical Vapor Deposition of a 20 -nm thick $\mathrm{Si}_{0.76} \mathrm{Ge}_{0.24}$ alloy layer on SOI (6 nm-thick Si layer on $20 \mathrm{~nm}$-thick $\mathrm{SiO}_{2}$ layer). The first step of the condensation process consists of a Rapid Thermal Oxidation performed at $1050^{\circ} \mathrm{C}$ for $230 \mathrm{~s} .{ }^{33}$ This process leads to the preferential oxidation of $\mathrm{Si}$ atoms and due to the very small miscibility of Ge in $\mathrm{SiO}_{2}$, the remaining $\mathrm{Ge}$ atoms are rejected into the underlying Si layer. Then, thermal oxide is removed before the deposition of $4 \mathrm{~nm}$-thick tetraethyl orthosilicate (TEOS). Then, when applicable, $55 \mathrm{~nm}$-thick silicon nitride is deposited by low pressure chemical vapor deposition (LPCVD). It is continued by a temperature annealing at $1050{ }^{\circ} \mathrm{C}$ during a longer time (30 min.) that allows Si and Ge interdiffusion and homogenisation of the SiGe alloy. Due to this thermal budget, the initial thin Si layer of the SOI substrate can be completely transformed, leading to a quasi-homogeneous Ge enriched SiGe layer directly on an insulator. It explains why the remaining Ge atoms are rejected into the underlying SiGe layer and how a quasi-homogeneous Ge-enriched SiGe layer can be obtained. ${ }^{31,34}$

The crystallization process maintains the strain imposed by the initial $\mathrm{Si} / \mathrm{SiO}_{2}$ interface and does not increase the number of structural extended defects. Hence, the condensed SiGe layer has a very good crystallinity with a high degree of control of the strain and composition. ${ }^{6,31,32,35-37}$ From the bottom thick Si substrate, the complete stacking of the samples studied in this paper consists of $20 \mathrm{~nm}$-thick $\mathrm{SiO}_{2}$ Buried-Oxide (the initial BOX of the SOI), 20 or 13 nm-thick $\mathrm{Si}_{0.76} \mathrm{Ge}_{0.24}, 4$ nm-thick $\mathrm{SiO}_{2}$ and an optional 55 nm-thick $\mathrm{SiN}_{x}$ layer.

In this letter, we study the individual strain relaxation of square patterns obtained by UV-lithography from strained SGOI layers. Three samples were synthesized at CEA-Leti and STMicroelectronics using a mask including SiGe lines and square or rectangular SiGe islands of lateral size $0.25,0.5,2$ and $5 \mu \mathrm{m}$, with an inter-spacing of the order of the object sizes (see Figure S2 in the supplementary materials for examples giving some examples of scanning electron microscopy images). The thickness of the SiGe patterns was:

- sample A: $20 \mathrm{~nm}$-thick SGOI with $55 \mathrm{~nm} \mathrm{SiN}_{x}$ on top

- sample B: 20 nm-thick SGOI without $\operatorname{SiN}_{x}$

- sample C: 13 nm-thick SGOI without $\mathrm{SiN}_{x}$ 
Note that such thickness for single SiGe nanostructures represents a challenge for X-ray measurements due to their quite small atomic scattering factors $\left(\mathrm{Z}_{S i}=14, \mathrm{Z}_{G e}=32\right) .{ }^{38}$

\section{EXPERIMENT}

We carried out the high resolution x-ray micro-diffraction measurements at the ID01 beamline $(\mathrm{ESRF})^{39}$. To be able to measure very thin nanostructures with a high lateral resolution, a specific setup has been used with a multilayer monochromator, but with a $0.31 \%$ bandwidth pink-beam illumination centered at $8 \mathrm{keV}$. The x-ray beam was then focused down to a $170 \mathrm{~nm}$ (vertical) x $220 \mathrm{~nm}$ (horizontal) spot size using a Kirkpatrick-Baez mirror (KB) in order to reach $3.5 \times 10^{6}$ photons $/ \mathrm{sec} / \mathrm{nm}^{2}$. Such a high flux is made possible because of the pink-beam illumination and enables unprecedented imaging of ultra-thin layer. The diffracted intensity is recorded by a fast readout two-dimensional photon counting detector with $516 \times 516$ pixels of $55 \mu \mathrm{m}$ size positioned at $96 \mathrm{~cm}$ from the sample. ${ }^{40}$ The sample was mounted on a fast scanning piezoelectric stage with a $2 \mathrm{~nm}$ resolution (see Figure $\mathrm{S} 3$ in the supplementary materials for the SXDM description and sketch of the experimental setup).

A rough alignment was first carried out by mapping out the etched patterns with an optical microscope mounted vertically on top of the sample. Then, a finer alignment was made possible by recording the diffracted intensity on the detector with the fast three dimensional SXDM at the Bragg peak: while raster scanning a zone of the sample, the intensity on the detector is summed for each scan point leading to an intensity map which gives a clear location of the features of interest. The real space scans were typically made over areas of about $10 \mu \mathrm{m}$ x $10 \mu \mathrm{m}$ with a $100 \mathrm{~nm}$ step size in both in-plane directions. The SiGe and Si Bragg angles were determined by making standard radial scans, taking advantage of slightly different SiGe and Si crystal orientations, both .

The $\mathbf{0 0 4}$ reflection of the buried $\mathrm{Si}$ (incidence angle of $34.8^{\circ}$ ) provides an absolute reference value for the further calculated lattice parameters. By identifying the coordinates of the center of mass of the Si Bragg peak on the detector, combined with the knowledge of the position of the direct beam and of the angles of the diffractometer, we measured $\mathrm{a}_{S i}=$ $4 \lambda /(2 \sin (\theta))=5.441 \AA$ which is within $0.19 \%$ relative error compared to the Si bulk lattice parameter tabulated at room temperature $\left(\mathrm{a}_{\mathrm{Si}}=5.431 \AA\right)$. Note that the approximation made by rounding the pixel coordinate corresponds to an accuracy of $0.005 \%$ relative to 
the tabulated Si lattice parameter. The 004 SiGe Bragg peak, which has a larger lattice parameter than $\mathrm{Si}\left(\mathrm{a}_{\mathrm{Ge}}=5.658 \AA\right)$, appears at a lower incident angle, i.e. at a lower scattering vector value $\left(\mathrm{Q}=4.589 \AA^{-1}\right)$. The two-dimensional scan around this reciprocal space point gives a real space distribution of the scattered intensity for the (004) SiGe planes, but to separate strain and tilt, we need to identify the position of the Bragg peak in the 3D reciprocal space.

Taking into account the spectral divergence of the pink-beam, this is achieved by a $0.4^{\circ}$ rotation of incident angles between $34.28^{\circ}\left(\mathbf{Q}=4.568 \AA^{-1}\right)$ and $34.68^{\circ}\left(\mathbf{Q}=4.614 \AA^{-1}\right)$ with a $0.01^{\circ}$ angular step. The same method was used to analyze the asymmetric SiGe (113) reflection, which is better separated from the contribution of the Si substrate compared to the (004) reflection, and has also the advantage to be almost tangential to the Ewald sphere (i.e. the information about the displacement field is contained in a single image). In order to acquire strain and tilt information from the $\mathbf{1 1 3}$ reflection, 3D scanning is performed for incident angles varying between $53.99^{\circ}$ and $54.39^{\circ}$ with a $0.01^{\circ}$ angular step, collecting the diffracted beam at $2 \theta=55.7^{\circ}$. Note that the $\mathbf{1 1 3}$ reflection also presents the advantage of being closer to a normal incidence on the sample, hence providing a smaller beam footprint (212 $\mathrm{nm}$ at $54^{\circ}$ against $300 \mathrm{~nm}$ at $34.5^{\circ}$ ) yielding higher spatial resolution in the vertical direction.

\section{RESULTS AND DISCUSSION}

\section{A. Comparison of growth methods}

As a reference used in further lithographic processes, the full-sheet strain corresponding to the initial SiGe layer obtained by condensation is measured on $50 \mu \mathrm{m}$-wide lines with $10 \mu \mathrm{m} \times 10 \mu \mathrm{m}$ area scan. The lateral scale of this pattern is indeed much larger than the usual elastic relaxation length induced by the edge boundaries as it will be demonstrated later. This measurement can be also compared to results gathered on a $70 \mathrm{~nm}$-thick SiGeon-SOI layer obtained by implantation, splitting and wafer transfer, corresponding to a completely different technological solution. Figure 1 shows the comparison of the different tilts measured from the 113 Bragg reflection on $50 \mu \mathrm{m}$-wide lines of $13 \mathrm{~nm}$-thick SiGe grown by condensation, i.e. sample $\mathrm{C}$ (first row: (a) to (c)), and on the $70 \mathrm{~nm}$-thick SiGe-on-SOI 
layer grown by smart-cut molecular bonding (second row: (a') to $\left(c^{\prime}\right)$ ). The displacement of the Bragg peak in the $2 \theta$ direction is directly related to the variation of the SiGe 113 lattice parameter - and hence closely related to the (004) lattice parameter as a full-sheet layer can be modeled with a biaxial approach:

$$
d_{113}=\left(2 / a_{[100]}^{2}+9 / a_{[001]^{2}}\right)^{-1 / 2}
$$

where $a_{[001]}$ denotes the out-of-plane lattice parameter and $a_{[100]}$ denotes the in-plane lattice parameter of the strained SiGe, which is equal to $a_{S i}$ for a fully-strained layer. Meanwhile, a transverse displacement of the Bragg peak is related to the orientation of the $\mathbf{1 1 3}$ reflection with respect to the [113] crystallographic direction.

Figures 1(b) and 1(c) (resp. 1(b') and 1(c')) show for the 13 nm-thick SiGe layer (resp. $70 \mathrm{~nm}$-thick SiGe layer) the tilt distributions taken at maximum intensity in the directions perpendicular and parallel to the scattering planes. The first row of Figure 1 shows that the tilt maps are very homogeneous with a standard deviation of both azimuthal and in-plane angle variations of about $\sigma_{a z \text { \&plan. }}=0.004^{\circ}$. It demonstrates that the growth-condensation process achieves dislocation and tilt-free layers. The same type of analysis performed with the $70 \mathrm{~nm}$-thick layer obtained by layer bonding and transfer is completely different. The mosaicity is about $0.10^{\circ}$ and the standard deviation of both azimuthal and planar tilt is $\sigma_{a z \text {.\&plan. }}=0.08^{\circ}$. This is explained by the occurrence of both horizontal and vertical stripes resulting from the standard cross-hatch pattern induced by the SiGe growth on Si created by $60^{\circ}$ misfit dislocations along the $<110>$ directions (a/2 [101] Burgers vector). ${ }^{21,41}$

\section{B. Quantitative maps}

Firstly, large patterns with $2 \times 2 \mu \mathrm{m}^{2} 20$ nm-thick SGOI squares with $\mathrm{SiN}_{x}$ on top (sample A) have been studied. Scanned areas of $12 \times 12 \mu \mathrm{m}^{2}$ have been performed with $100 \mathrm{~nm}$ step size in each direction of the piezo motors. As expected from the additional protection to x-rays provided by the Silicon Nitride, we checked that these patterns were very stable under the beam and we did not observe a significant evolution of the intensity during the measurement as observed in bare Si stripes. ${ }^{38}$ We have recorded both the 113 and 004 Bragg reflections analyzed in the first (resp. second) lines of Figure 2. The left hand side of Figure 2 depicts, for each pixel of the map, the $2 \mathrm{D}$ integrated intensity over the detector frame 


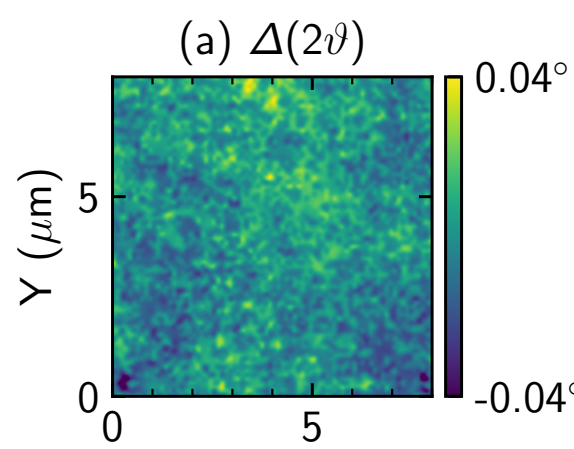

\section{(b) Azimuthal tilt}
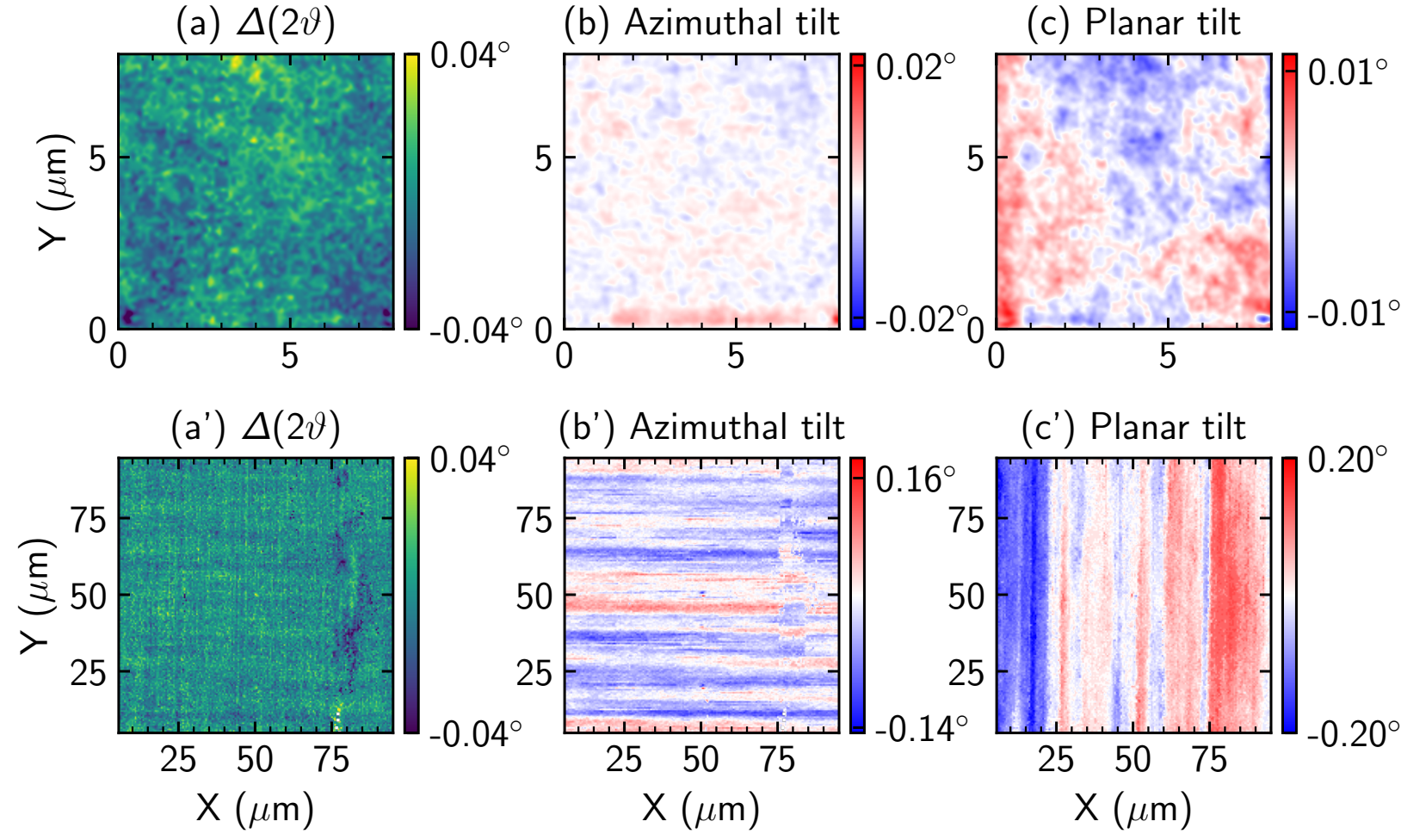

(b') Azimuthal tilt
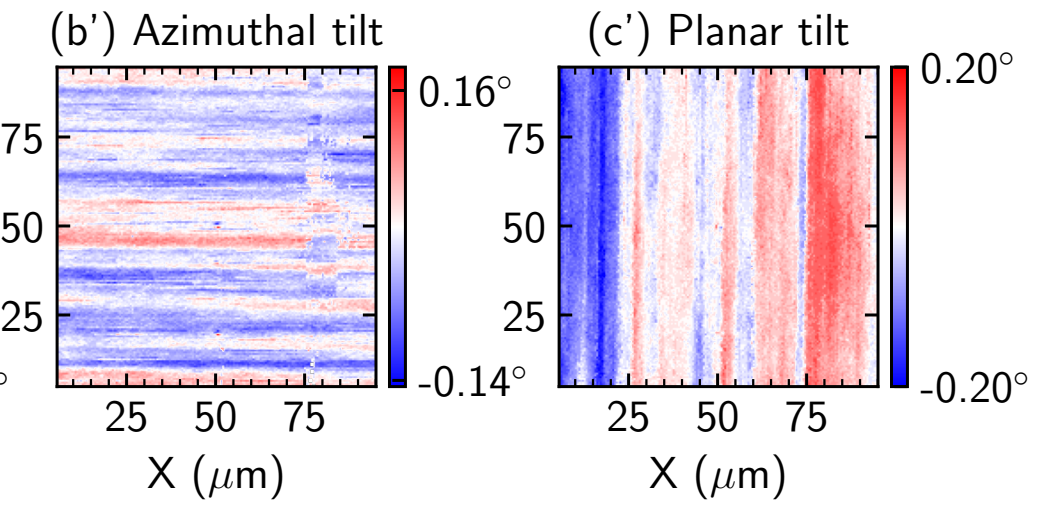

Figure 1. Comparison of the diffraction maps recorded on (a-c) a $13 \mathrm{~nm}$-thick SiGe layer obtained by condensation method (first row) and (a'-c') on a $70 \mathrm{~nm}$-thick sSOI layer obtained by traditional

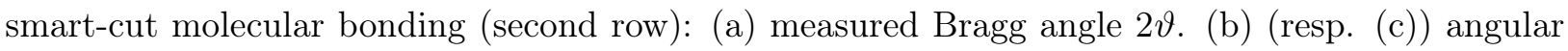
deviation of the diffracted beam in the direction perpendicular (resp. parallel) to the scattering plane. The sSOI sample (second line) shows a vertical defect positioned at about $\mathrm{X}=80 \mu \mathrm{m}$ and the vertical and horizontal stripes appearing on the tilt maps are related to the cross-hatch pattern. These stripes are not present in the condensation sample (note the different color scales for (b-b') and $\left.\left(c-c^{\prime}\right)\right)$, demonstrating the effectiveness of the condensation method to lower tilt fluctuations.

corresponding to the most intense slice of the SiGe Bragg peak. Note that the integration procedure includes masking the areas where the substrate peak is diffraction (including its crystal truncation rod), which is essential as the intensity is very low for each individual frame, as depicted in supplementary figure S4.

This maximum intensity map is taken as reference to determine a threshold, which enables to separate the squared islands from the substrate underneath, generating the white masks of the other maps. Note that the squares appear less sharp for the $\mathbf{0 0 4}$ measurements as compared with the ones from 113 measurements, because for this data acquisition geometry: 
(a) I Obs

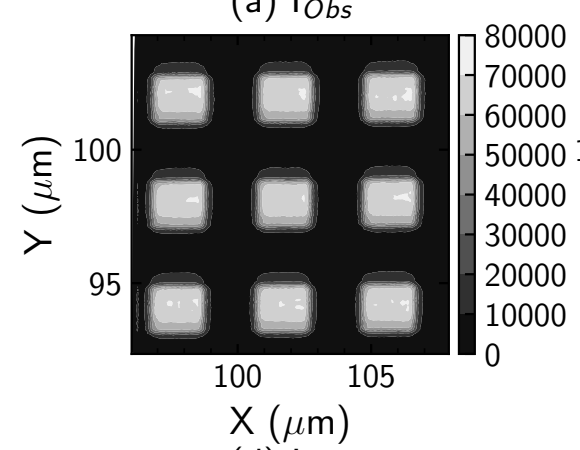

(d) $I_{O b s}$

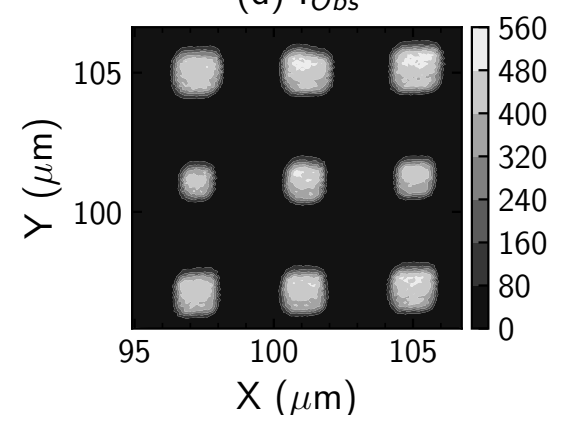

(b) RMS tilt

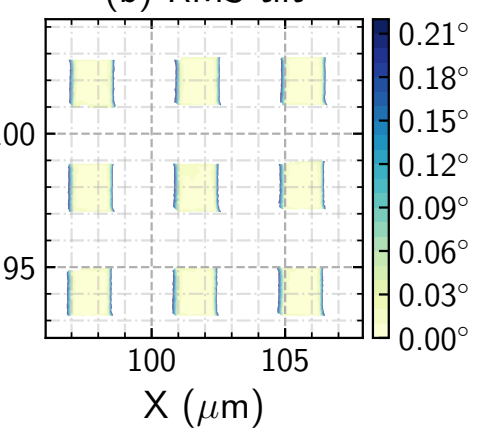

(e) RMS tilt

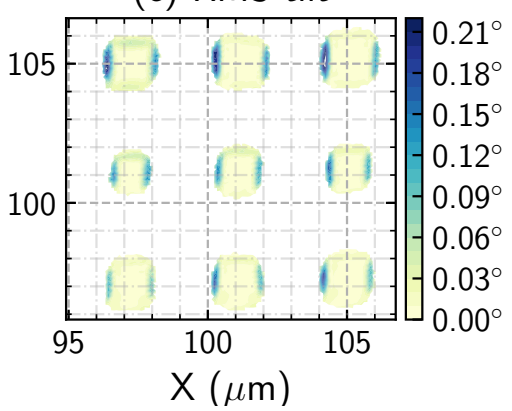

(c) $\epsilon_{113}=\left(a-a_{S i}\right) / a_{S i}$

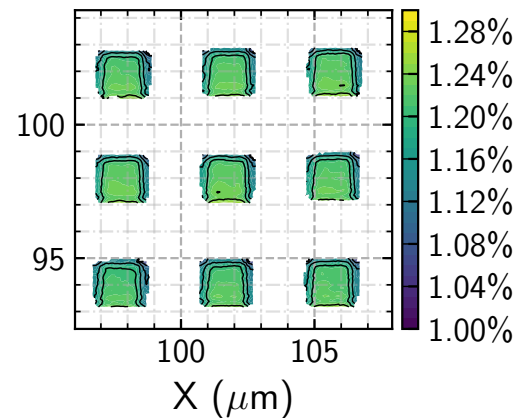

(f) $\epsilon_{004}=\left(a-a_{S i}\right) / a_{S i}$

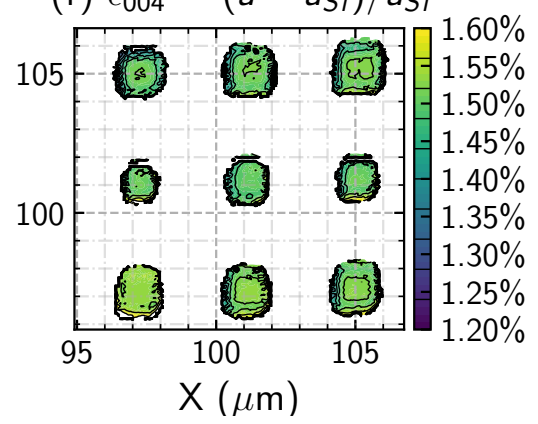

Figure 2. Map of a $12 \times 12 \mu \mathrm{m}^{2}$ area of strained SiGe-on-insulator $2 \mathrm{x} 2 \mu \mathrm{m}^{2}$ islands of thickness $20 \mathrm{~nm}$ (sample A), for both 113 (a-c) and 004 (d-f) Bragg reflections. (a,d) Maximum of intensity integrated on the whole detector for each pixel. (b,e) RMS tilt angle, considering both the perpendicular and parallel angular deviation with respect to the scattering plane showing a clear evidence of the side relaxation of the islands. (f) $\epsilon_{z z}$ out-of-plane deformation distribution, (c) $\epsilon_{113}$ deformation distribution, both calculated with respect to the bulk Si lattice parameter. The incident and diffracted beams are in the XZ plane. The slightly smaller size of the islands for the (004) reflections in (d-f) is due to the larger relative deformation, which reduces the intensity on the borders. Some interface defects may explain the differences in the middle horizontal island sizes.

(i) the silicon substrate peak is closer to the SiGe (i.e. the overlap is higher) and (ii) the beam footprint on the sample is wider (it causes a blurring effect because the incident angles are respectively $54^{\circ}$ and $34.5^{\circ}$ ). It is also important to stress that the quality of the results is poorer in the vertical (Y) direction due to the asymmetry of the incoming beam , reducing the spatial resolution in this direction.

The consequence of which was the expected four-fold symmetry of deformation and tilt is reduced to a two-fold horizontal symmetry. Indeed, the lattice tilts of the SiGe layer are due to the elastic relaxation at the free boundary conditions introduced by sidewall etching, 
considering that the bottom of this layer is still compressed by the substrate. Maps at the middle of Figure 2 show the root mean square (RMS) of both azimuthal and planar tilts. The sidewall relaxation of the squares induces up to $0.26^{\circ} \mathrm{RMS}$ tilt for the (113) lattice planes, and $0.18^{\circ}$ RMS tilt for the (004) lattice planes. Furthermore, the out-of-plane $(004)$ deformation $\left(\epsilon_{(004)}\right)$ and the (113) deformation - mixing in-plane and out-of-plane are depicted in the right-hand side of Figure 2. In this paper, deformation ${ }^{42}$ is defined with respect to the Si lattice parameter, i.e., for a given (hkl) reflection:

$$
\epsilon_{h k l}=\frac{a_{h k l} \sqrt{h^{2}+k^{2}+l^{2}}}{a_{S i}}-1
$$

We observe a good homogeneity of both deformations at the center of the squares and a small variation at the edges of the squares due to sidewall relaxation.

Secondly, smaller patterns with 500x500 nm² $13 \mathrm{~nm}$-thick SGOI squares without $\mathrm{SiN}_{x}$ on top (sample C) have been analyzed. Although this sample contains a very low amount of $\mathrm{SiGe}$, we were still able to discern the patterns and isolate their diffraction spots. Figure 3 shows the intensity, RMS tilt and deformation maps from a $8 \times 8 \mu \mathrm{m}^{2}$ area scan at the $\mathbf{1 1 3}$ Bragg reflection condition. One can note a RMS tilt of $0.07^{\circ}$ on the edges of the patterns and especially an homogeneous deformation across the area, with variations smaller than $0.05 \%$. This suggests that the scanned area is made of patterns that are all close to their completely relaxed state and also provides an estimation of the relaxation length of the compressive in-plane deformation that must be at least of the same order of magnitude as half the width of the measured patterns $(>250 \mathrm{~nm})$.

\section{Statistical information}

SXDM allows us not only to study the homogeneity of the sSGOI patterns, but also to gather statistical information. For instance, an interesting feature that can be extracted from the data is the mean value of deformation at the center of each pattern. By defining an integration zone of $300 \times 300 \mathrm{~nm}^{2}$ centered on each pattern, corresponding to $3 \times 3$ points of the map, one can compare the mean deformation over multiple patterns. These central areas are supposed to be homogeneous unlike the edges, where the introduction of free boundaries by etching modifies the strain state.

Figure 4 shows the histograms extracted from the $2 \mathrm{x} 2 \mu \mathrm{m}^{2}$ patterns of sample A \& B 

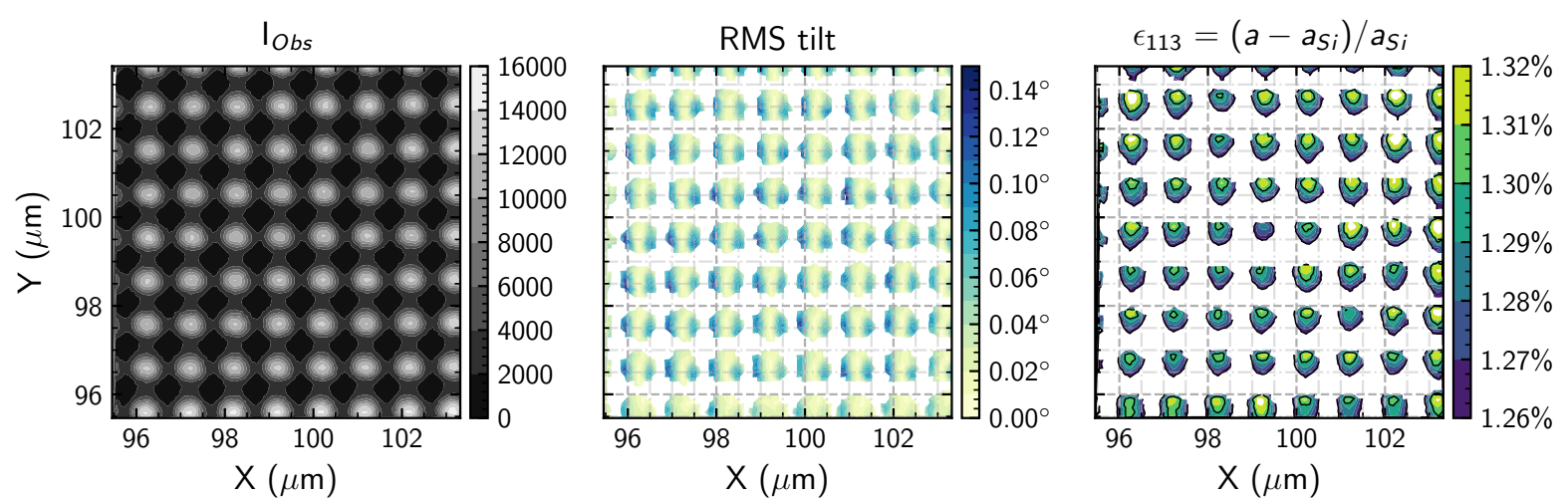

Figure 3. Mapping of a $8 \times 8 \mu \mathrm{m}^{2}$ area of strained SiGe-on-insulator $500 \times 500 \mathrm{~nm}^{2}$ square islands of thickness $20 \mathrm{~nm}$ (sample A) for the 113 Bragg reflection. (Left) Maximum of intensity integrated on the whole detector for each pixel. (Center) RMS tilt angle, taking into account both the perpendicular and parallel angular deviation with respect to the scattering plane. (Right) $\epsilon_{113}$ deformation distribution, both calculated with respect to the bulk Si lattice parameter. For these small islands, the $170 \mathrm{~nm}(\mathrm{v}) \times 220 \mathrm{~nm}(\mathrm{~h})$ size of the beam limits the resolution, so we focused the quantitative analysis to the deformation at the center of the islands, as shown in Fig. 4

and from the $500 \times 500 \mathrm{~nm}^{2}$ patterns of sample C. It is striking that there is a very small deviation around the mean value for samples A \& B, calculated as the RMS of the statistical distribution of extracted deformation: $\pm 0.004 \%$ for sample A and $\pm 0.007 \%$ for sample B. Then, sample C presents a RMS of $\pm 0.03 \%$. This increase is likely to be a consequence of the smaller thickness of the sample, making the growth process more sensitive. Note also that the initial wafer is different for every sample. Overall, these small variations outline the very good homogeneity over one given wafer. Next, the tensile role of the nitride cap is shown by comparing sample A and sample B. For the same $2 \times 2 \mu \mathrm{m}^{2}$ pattern, the mean (113) deformation is lower in the absence of $\operatorname{SiN}_{x}$ (1.125\% without, $1.227 \%$ with). As the (113) deformation is mainly proportional to the out-of-plane strain, and the layers supposed to be biaxially strained, a lower (113) deformation is linked to a larger relaxation of the inplane strain. Hence, the intrinsically stressed $\operatorname{SiN}_{x}$ layer helps maintaining the compressive in-plane strain. 


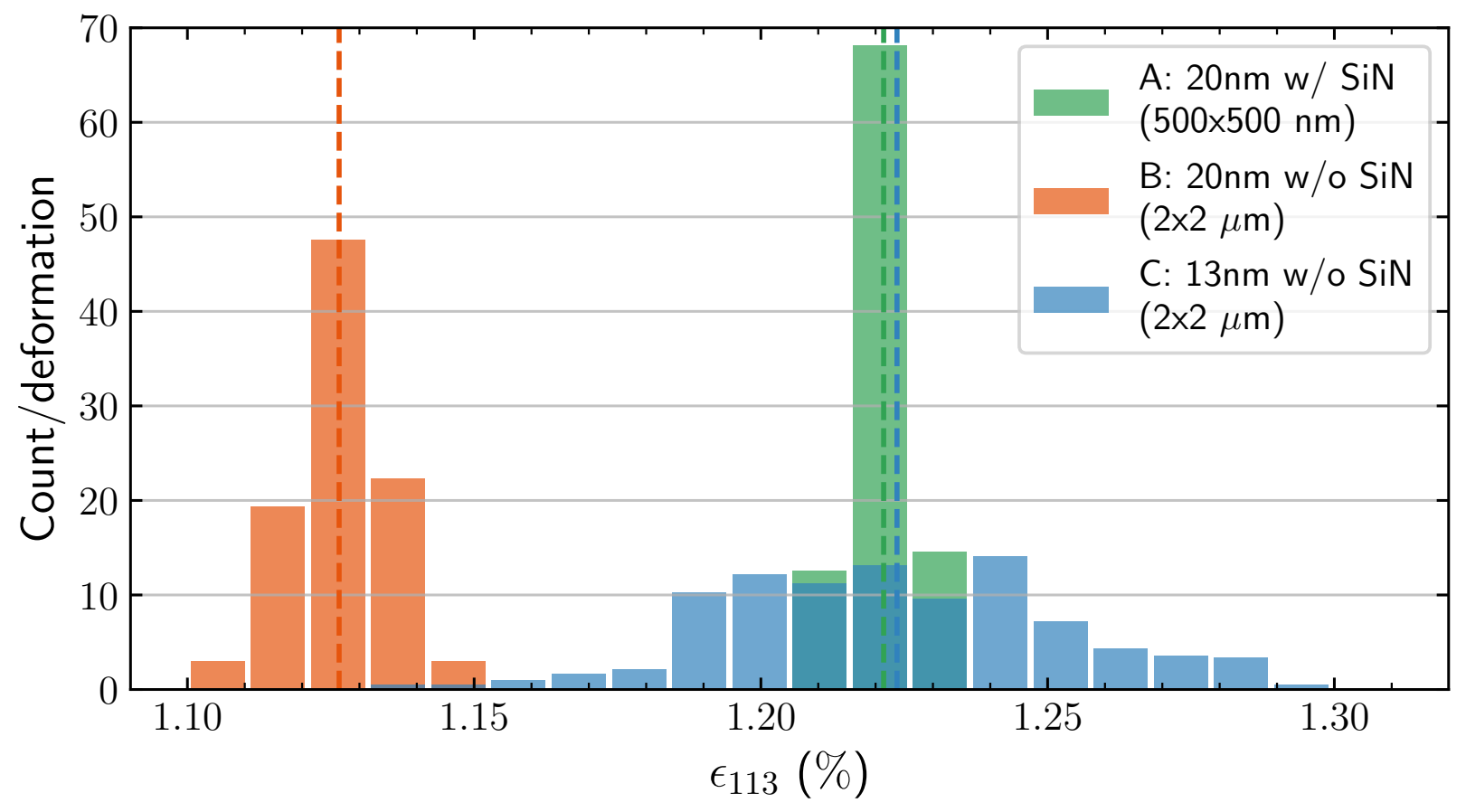

Figure 4. Distribution of (113) deformation from the central areas of all the probed patterns on each sample. The dotted lines depict the average deformation over the entire distribution. The bar histograms represent the density of the distribution, i.e., a number of counts by deformation value. The mean value and RMS for each sample are the following: $1.125 \% \pm 0.004 \%$ for sample A $(2 \times 2$ $\left.\mu \mathrm{m}^{2}\right), 1.227 \% \pm 0.007 \%$ for sample B $\left(2 \mathrm{x} 2 \mu \mathrm{m}^{2}\right)$ and $1.224 \% \pm 0.03 \%$ for sample $\mathrm{C}\left(0.5 \mathrm{x} 0.5 \mu \mathrm{m}^{2}\right)$.

\section{Relaxation lengths}

Finally, it is possible to assess the relaxation at the edges of the patterns. To do so, we extracted the deformation profiles of the largest patterns from the $13 \mathrm{~nm}$-thick sSGOI sample. The choice of sample was driven by the fact that the mean value of the $\epsilon_{113}$ is the same for the $2 \mathrm{x} 2 \mu \mathrm{m}^{2}$ patterns and the $5 \mathrm{x} 5 \mu \mathrm{m}^{2}$ patterns. Figure 5 presents the deformation profiles (dots symbols) along with an empirical model (straight lines) adapted from Ref. 43 in order to derive a typical relaxation length. The fit function, based on an exponential decay, is the following:

$$
f_{\text {relax }}=f_{\text {min }}+\left(f_{\text {max }}-f_{\text {min }}\right)\left[\left(1-e^{\frac{-x}{\lambda}}\right)-e^{\frac{x-L_{a c t}}{\lambda}}\right]
$$


where $f_{\min }$ and $f_{\max }$ are the minimum and maximum deformation values, $L_{a c t}$ the width of the pattern and $\lambda$ the relaxation length (see Table T1 in the supplementary materials for the fitted values of the four parameters). The $2 \times 2 \mu \mathrm{m}^{2}$ patterns has an average value of $\lambda$ of about $384 \mathrm{~nm}$ with a variation from 333 to $430 \mathrm{~nm}$, whereas the relaxation length of the $5 \mathrm{x} 5 \mu \mathrm{m}^{2}$ patterns has smaller variation and is close to $301 \mathrm{~nm}$.

We have also included in Fig. 5 the relaxation curves simulated using Finite Element Modeling (FEM): the main results from this elasticity modeling are that the relaxation lengths are slightly smaller for the FEM data - which could be due to the broadening due to the beam size -, and that the relaxation on the island borders is significantly larger experimentally compared to the elastic model. ${ }^{44}$

Such relaxation determined from X-ray diffraction has the same order of magnitude as what has been determined by electron microscopy on similar samples. An $\epsilon_{x x}$ profile has been determined by both; nano-beam electron diffraction (NBED) to extend spatially up to $400 \mathrm{~nm}^{43}$, and Dark-Field Electron Holography (DFEH) up to more than $300 \mathrm{~nm} \cdot{ }^{37}$ Note also that these techniques have also to take into account carefully the lattice rotations to get precise measurements of the deformations. An interface glide at the edge of the pattern has been proposed that could result from the two of the main steps of the technological process: the heteroepitaxy of SiGe on an SOI substrate and the rapid thermal oxidation condensation and annealing. This interface gliding could explain the larger relaxation in the experimental data compared to the elastic model.

\section{CONCLUSION}

In conclusion, we showed that the SXDM technique yields accurate and precise quantitative deformation measurements of ultra-thin SiGe layers whose diffraction can be shadowed by intense substrate scattering. From these quantitative deformation maps, we were able to evaluate the reliability of the condensation technique used to integrate strain in FDSOI. In comparison with the traditional implantation, smart-cut, and bonding technique, it appears that the condensation process not only removes the cross-hatch patterning but also yields less disoriented layers.

Furthermore, we observed that the strain over the central area of patterns with a given width is homogeneous, with relative variations of less than $1.2 \%$ for the $20 \mathrm{~nm}$-thick samples, 

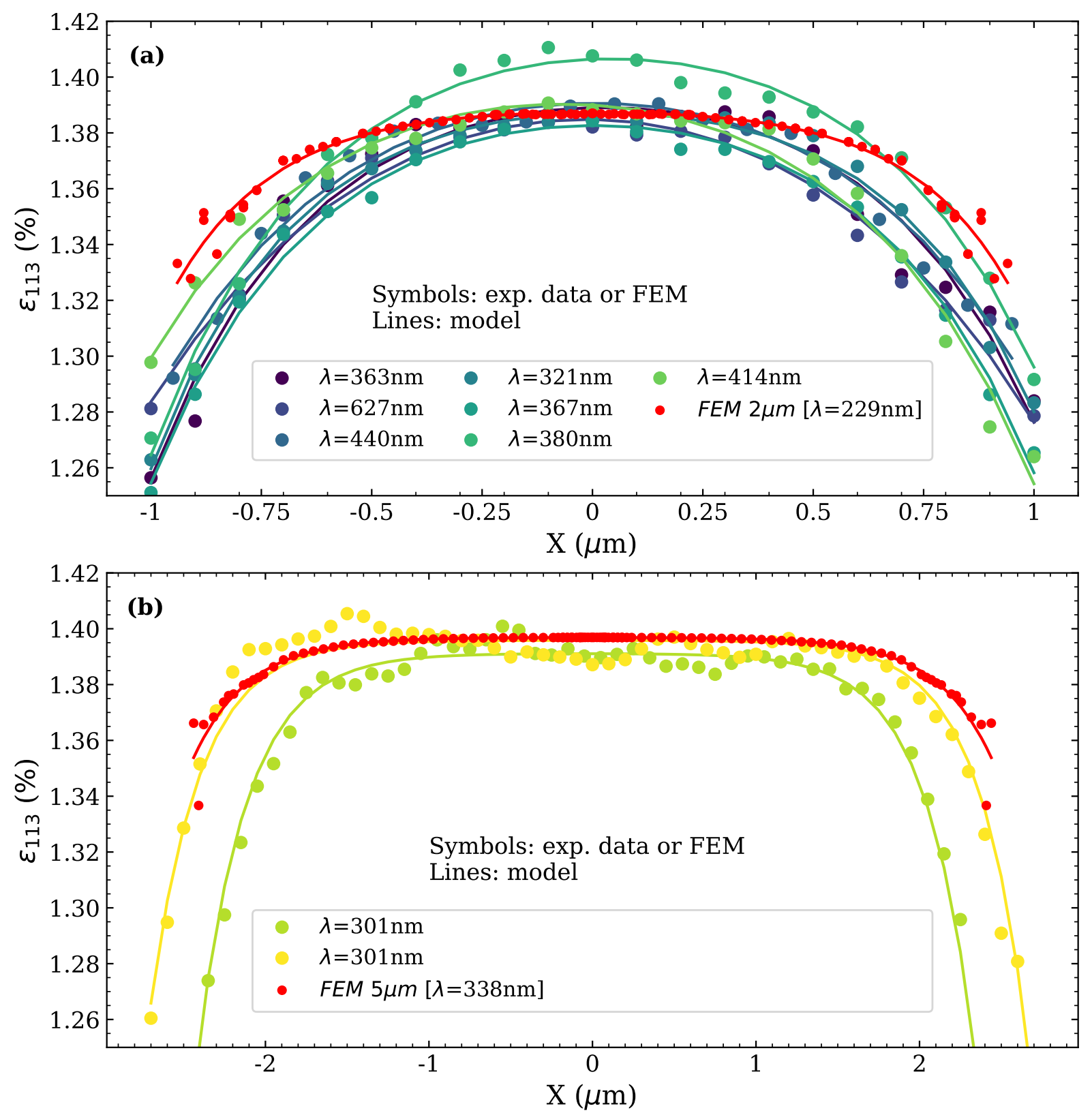

Figure 5. (113) deformation profiles from (a) $2 \times 2 \mu \mathrm{m}^{2}$ patterns (6 islands) and (b) $5 \times 5 \mu \mathrm{m}^{2}$ (two different islands) of $13 \mathrm{~nm}$-thick SGOI (sample C) and corresponding curves from the empirical model (see equation 3). The typical relaxation lengths $\lambda$ obtained with the analytical model (lines) are in agreement with the data (dots) and are between $234 \mathrm{~nm}$ and $377 \mathrm{~nm}$. Uncertainties on deformations values are estimated to $\pm 0.002 \%$. The red curves correspond to FEM elasticity models, which present slightly smaller relaxation lengths, but significantly less relaxation on the border of the islands.

and of $4.9 \%$ for the $13 \mathrm{~nm}$-thick sample. Also, by measuring that the (113) strain of the 20 
nm-thick sample with $\mathrm{SiN}_{x}$ on top is larger than the one of the $20 \mathrm{~nm}$-thick sample without $\mathrm{SiN}_{x}$, we showed that a tensile stressed $\mathrm{SiN}_{x}$ cap layer helps to maintain the compressive in-plane strain of the SiGe layer.

Finally, this study also gave insights on the typical lattice rotation and relaxation length of the in-plane strain, caused by the introduction through etching, of free boundaries on the side of the patterns. A comparison with elasticity models shows that while the measured relaxation lengths are generally between 300 and $400 \mathrm{~nm}$, the simulated ones are slightly smaller. The most striking difference is that the amplitude of the measured relaxation on the border is much larger than predicted by the simulations, probably due to non-elastic processes. Indeed, the fact that the mean strains on the central area of all patterns with width above $500 \mathrm{~nm}$ are equal and higher than the ones from all patterns with width below $500 \mathrm{~nm}$, demonstrates that the smallest patterns are not fully strained. Moreover, estimated relaxation lengths were extracted from strain profiles along the width of several patterns. Interestingly, these estimated values are in agreement with recent electron holography measurements which tend to validate the assumption of an interface glide at the boundary between the SiGe and the BOX.37

\section{SUPPLEMENTARY MATERIAL}

See supplementary material for the (i) description of the samples with a schematic of the condensation process (S1), scanning electron microscopy images of patterned samples (S2), a sketch of the experimental setup (S3), an example of diffraction frame exhibiting the low intensity counts (S4), and the results of the fits of Figure 5 (T1).

\section{ACKNOWLEDGEMENTS}

Authors would like to acknowledge the ESRF ID01 beamline for the beamtimes MA2625 and MA2242, as well as STMicroelectronics for the preparation of samples. The PhD scholarship of GG has been partially granted by the French ANR Labex LANEF (10-LABX-0051). 


\section{DATA AVAILABILITY STATEMENT}

Raw data were generated at the European Synchrotron Radiation Facility (ESRF). Derived data supporting the findings of this study are available from the corresponding author upon reasonable request.

\section{REFERENCES}

${ }^{1}$ D. J. Dunstan, "Strain and strain relaxation in semiconductors," Journal of Materials Science: Materials in Electronics 8, 337-375 (1997).

${ }^{2}$ M. L. Lee, E. A. Fitzgerald, M. T. Bulsara, M. T. Currie, and A. Lochtefeld, "Strained $\mathrm{Si}, \mathrm{SiGe}$, and Ge channels for high-mobility metal-oxide-semiconductor field-effect transistors," J. Appl. Phys. 97, 011101-011101-28 (2005).

${ }^{3}$ S. Bedell, A. Khakifirooz, and D. Sadana, "Strain scaling for CMOS," MRS Bulletin 39, 131-137 (2014), publisher: Cambridge University Press.

${ }^{4}$ D. J. Paul, "Silicon-Germanium Strained Layer Materials in Microelectronics," Advanced Materials 11, 191-204 (1999),_eprint: https://onlinelibrary.wiley.com/doi/pdf/10.1002/\%28SICI\%2915214095\%28199903\%2911\%3A3\%3C191\%3A\%3AAID-ADMA191\%3E3.0.CO\%3B2-3.

${ }^{5}$ S. Baudot, F. Andrieu, O. Faynot, and J. Eymery, "Electrical and diffraction characterization of short and narrow MOSFETs on fully depleted strained silicon-on-insulator (sSOI)," Solid-State Electron. 54, 861-869 (2010).

${ }^{6}$ M. Casse, L. Hutin, C. Le Royer, D. Cooper, J.-M. Hartmann, and G. Reimbold, "Experimental Investigation of Hole Transport in Strained $\$ \backslash h b o x\{\mathrm{Si}\}_{-}\{1$ $\mathrm{x}\} \backslash \mathrm{hbox}\{\mathrm{Ge}\}_{-}\{\mathrm{x}\} / \triangle$ hbox $\{\mathrm{SOI}\} \$$ pMOSFETs_Part I: Scattering Mechanisms in LongChannel Devices," IEEE Trans. Electron Devices 59, 316-325 (2012).

${ }^{7}$ O. Weber, E. Josse, J. Mazurier, N. Degors, S. Chhun, P. Maury, S. Lagrasta, D. Barge, J. Manceau, and M. Haond, "14nm FDSOI upgraded device performance for ultra-low voltage operation," in 2015 Symposium on VLSI Technology (VLSI Technology) (2015) pp. T168-T169.

${ }^{8}$ K. Cheng, A. Khakifirooz, N. Loubet, S. Luning, T. Nagumo, M. Vinet, Q. Liu, A. Reznicek, T. Adam, S. Naczas, P. Hashemi, J. Kuss, J. Li, H. He, L. Edge, J. Gimbert, 
P. Khare, Y. Zhu, Z. Zhu, A. Madan, N. Klymko, S. Holmes, T. M. Levin, A. Hubbard, R. Johnson, M. Terrizzi, S. Teehan, A. Upham, G. Pfeiffer, T. Wu, A. Inada, F. Allibert, B.-Y. Nguyen, L. Grenouillet, Y. Le Tiec, R. Wacquez, W. Kleemeier, R. Sampson, R. H. Dennard, T. H. Ning, M. Khare, G. Shahidi, and B. Doris, "High performance extremely thin SOI (ETSOI) hybrid CMOS with Si channel NFET and strained SiGe channel PFET," in 2012 International Electron Devices Meeting (IEEE, San Francisco, CA, USA, 2012) pp. 18.1.1-18.1.4.

${ }^{9}$ S. Baudot, F. Andrieu, O. Weber, P. Perreau, J.-F. Damlencourt, S. Barnola, T. Salvetat, L. Tosti, L. Brevard, D. Lafond, J. Eymery, and O. Faynot, "Fully Depleted Strained Silicon-on-Insulator p-MOSFETs With Recessed and Embedded Silicon-Germanium Source/Drain," IEEE Electron Device Lett. 31, 1074-1076 (2010).

${ }^{10}$ F. Pezzoli, E. Bonera, E. Grilli, M. Guzzi, S. Sanguinetti, D. Chrastina, G. Isella, H. von Känel, E. Wintersberger, J. Stangl, and G. Bauer, "Raman spectroscopy determination of composition and strain in heterostructures," Materials Science in Semiconductor Processing 11, 279-284 (2008).

${ }^{11}$ L. H. Wong, C. C. Wong, J. P. Liu, D. K. Sohn, L. Chan, L. C. Hsia, H. Zang, Z. H. Ni, and Z. X. Shen, "Determination of Raman Phonon Strain Shift Coefficient of Strained Silicon and Strained SiGe," Jpn. J. Appl. Phys. 44, 7922-7924 (2005).

${ }^{12}$ S. Nakashima, T. Mitani, M. Ninomiya, and K. Matsumoto, "Raman investigation of strain in $\mathrm{Si} / \mathrm{SiGe}$ heterostructures: Precise determination of the strain-shift coefficient of Si bands," Journal of Applied Physics 99, 053512 (2006).

${ }^{13}$ S. C. Jain, B. Dietrich, H. Richter, A. Atkinson, and A. H. Harker, "Stresses in strained GeSi stripes: Calculation and determination from Raman measurements," Phys. Rev. B 52, 6247-6253 (1995).

${ }^{14}$ B. Dietrich, E. Bugiel, H. J. Osten, and P. Zaumseil, "Raman investigations of elastic strain relief in $\mathrm{Si}{ }_{1-x} \mathrm{Ge}{ }_{x}$ layers on patterned silicon substrate," Journal of Applied Physics 74, 7223-7227 (1993).

${ }^{15}$ M. Takei, D. Kosemura, K. Nagata, H. Akamatsu, S. Mayuzumi, S. Yamakawa, H. Wakabayashi, and A. Ogura, "Channel strain analysis in high-performance damascene-gate p-metal-oxide-semiconductor field effect transistors using high-spatial resolution Raman spectroscopy," Journal of Applied Physics 107, 124507 (2010). 
${ }^{16}$ M. Gailhanou, A. Loubens, J.-S. Micha, B. Charlet, A. A. Minkevich, R. Fortunier, and O. Thomas, "Strain field in silicon on insulator lines using high resolution x-ray diffraction," Appl. Phys. Lett. 90, 111914 (2007).

${ }^{17}$ A. A. Minkevich, M. Gailhanou, J.-S. Micha, B. Charlet, V. Chamard, and O. Thomas, "Inversion of the diffraction pattern from an inhomogeneously strained crystal using an iterative algorithm," Phys. Rev. B 76, 104106-5 (2007).

${ }^{18}$ C. E. Murray, H.-F. Yan, I. C. Noyan, Z. Cai, and B. Lai, "High-resolution strain mapping in heteroepitaxial thin-film features," Journal of Applied Physics 98, 013504 (2005).

${ }^{19}$ C. E. Murray, A. Ying, S. M. Polvino, I. C. Noyan, M. Holt, and J. Maser, "Nanoscale silicon-on-insulator deformation induced by stressed liner structures," J. Appl. Phys. 109, 083543 (2011).

${ }^{20}$ G. A. Chahine, M.-I. Richard, R. A. Homs-Regojo, T. N. Tran-Caliste, D. Carbone, V. L. R. Jacques, R. Grifone, P. Boesecke, J. Katzer, I. Costina, H. Djazouli, T. Schroeder, and T. U. Schülli, "Imaging of strain and lattice orientation by quick scanning X-ray microscopy combined with three-dimensional reciprocal space mapping," Journal of Applied Crystallography 47, 762-769 (2014).

${ }^{21}$ M. H. Zoellner, M.-I. Richard, G. A. Chahine, P. Zaumseil, C. Reich, G. Capellini, F. Montalenti, A. Marzegalli, Y.-H. Xie, T. U. Schülli, M. Häberlen, P. Storck, and T. Schroeder, "Imaging Structure and Composition Homogeneity of $300 \mathrm{~mm}$ SiGe Virtual Substrates for Advanced CMOS Applications by Scanning X-ray Diffraction Microscopy," ACS Appl. Mater. Interfaces 7, 9031-9037 (2015).

${ }^{22}$ S. O. Hruszkewycz, M. V. Holt, C. E. Murray, J. Bruley, J. Holt, A. Tripathi, O. G. Shpyrko, I. McNulty, M. J. Highland, and P. H. Fuoss, "Quantitative Nanoscale Imaging of Lattice Distortions in Epitaxial Semiconductor Heterostructures Using Nanofocused X-ray Bragg Projection Ptychography," Nano Lett. 12, 5148-5154 (2012).

${ }^{23}$ S. O. Hruszkewycz, M. V. Holt, M. Allain, V. Chamard, S. M. Polvino, C. E. Murray, and P. H. Fuoss, "Efficient modeling of Bragg coherent x-ray nanobeam diffraction," Opt. Lett., OL 40, 3241-3244 (2015).

${ }^{24}$ S. O. Hruszkewycz, M. Allain, M. V. Holt, C. E. Murray, J. R. Holt, P. H. Fuoss, and V. Chamard, "High-resolution three-dimensional structural microscopy by single-angle Bragg ptychography," Nat Mater 16, 244-251 (2016). 
${ }^{25}$ S. Baudot, F. Andrieu, F. Rieutord, and J. Eymery, "Elastic relaxation in patterned and implanted strained silicon on insulator," J. Appl. Phys. 105, 114302-10 (2009).

${ }^{26}$ D. Gu, M. Zhu, G. K. Celler, and H. Baumgart, "Size and Thickness Effect on the Local Strain Relaxation in Patterned Strained Silicon-on-Insulator," Electrochem. Solid-State Lett. 12, H113 (2009).

${ }^{27}$ O. Moutanabbir, M. Reiche, A. Hähnel, M. Oehme, and E. Kasper, "Multiwavelength micro-Raman analysis of strain in nanopatterned ultrathin strained silicon-on-insulator," Appl. Phys. Lett. 97, 053105 (2010).

${ }^{28}$ T. Tezuka, N. Sugiyama, T. Mizuno, M. Suzuki, and S.-i. Takagi, "A Novel Fabrication Technique of Ultrathin and Relaxed SiGe Buffer Layers with High Ge Fraction for Sub-100 nm Strained Silicon-on-Insulator MOSFETs," Jpn. J. Appl. Phys. 40, 2866-2874 (2001).

${ }^{29}$ T. Tezuka, S. Nakaharai, Y. Moriyama, N. Sugiyama, and S. Takagi, "High-mobility strained SiGe-on-insulator pMOSFETs with Ge-rich surface channels fabricated by local condensation technique," IEEE Electron Device Lett. 26, 243-245 (2005).

${ }^{30}$ T. Tezuka, S. Nakaharai, Y. Moriyama, N. Hirashita, E. Toyoda, T. Numata, T. Irisawa, K. Usuda, N. Sugiyama, T. Mizuno, and S.-i. Takagi, "Strained SOI/SGOI dual-channel CMOS technology based on the Ge condensation technique," Semicond. Sci. Technol. 22, S93-S98 (2007).

${ }^{31}$ T. David, A. Benkouider, J.-N. Aqua, M. Cabie, L. Favre, T. Neisius, M. Abbarchi, M. Naffouti, A. Ronda, K. Liu, and I. Berbezier, "Kinetics and Energetics of Ge Condensation in SiGe Oxidation," J. Phys. Chem. C 119, 24606-24613 (2015).

${ }^{32}$ V. Boureau, D. Benoit, B. Warot, M. Hÿtch, and A. Claverie, "Strain/composition interplay in thin SiGe layers on insulator processed by Ge condensation," Materials Science in Semiconductor Processing E-MRS Spring Meeting 2015 Symposium Z: Nanomaterials and processes for advanced semiconductor CMOS devices, 42, 251-254 (2016).

${ }^{33} \mathrm{~V}$. Boureau, Déformations introduites lors de la fabrication de transistors FDSOI : une contribution de l'holographie électronique en champ sombre, Ph.D. thesis, Univ. Toulouse, Toulouse, France (2016).

${ }^{34}$ F. Roze, O. Gourhant, E. Blanquet, F. Bertin, M. Juhel, F. Abbate, C. Pribat, and R. Duru, "Comparative Analysis of Growth Rate Enhancement and Ge Redistribution during Silicon-Germanium Oxidation by Rapid Thermal Oxidation," ECS Transactions 75, 67-78 (2016). 
${ }^{35}$ Y. Zhang, K. Cai, C. Li, S. Chen, H. Lai, and J. Kang, "Strain Relaxation in Ultrathin SGOI Substrates Fabricated by Multistep Ge Condensation Method," J. Electrochem. Soc. 156, H115 (2009).

${ }^{36}$ J. Suh, R. Nakane, N. Taoka, M. Takenaka, and S. Takagi, "Highly strained-SiGe-oninsulator p-channel metal-oxide-semiconductor field-effective transistors fabricated by applying Ge condensation technique to strained-Si-on-insulator substrates," Applied Physics Letters 99, 142108 (2011).

${ }^{37}$ V. Boureau, S. Reboh, D. Benoit, M. Hÿtch, and A. Claverie, "Strain evolution of SiGe-oninsulator obtained by the Ge-condensation technique," APL Materials 7, 041120 (2019).

${ }^{38}$ F. Mastropietro, J. Eymery, G. Carbone, S. Baudot, F. Andrieu, and V. Favre-Nicolin, "Time-Dependent Relaxation of Strained Silicon-on-Insulator Lines Using a Partially Coherent X-Ray Nanobeam," Phys. Rev. Lett. 111, 215502 (2013).

${ }^{39}$ S. J. Leake, G. A. Chahine, H. Djazouli, T. Zhou, C. Richter, J. Hilhorst, L. Petit, M.-I. Richard, C. Morawe, R. Barrett, L. Zhang, R. A. Homs-Regojo, V. Favre-Nicolin, P. Boesecke, and T. U. Schülli, "The Nanodiffraction beamline ID01/ESRF: a microscope for imaging strain and structure," Journal of Synchrotron Radiation 26, 571-584 (2019).

${ }^{40}$ C. Ponchut, J. M. Rigal, J. Clément, E. Papillon, A. Homs, and S. Petitdemange, "MAXIPIX, a fast readout photon-counting X-ray area detector for synchrotron applications," J. Instr. 6, C01069-C01069 (2011).

${ }^{41}$ H. Chen, Y. K. Li, C. S. Peng, H. F. Liu, Y. L. Liu, Q. Huang, J. M. Zhou, and Q.-K. Xue, "Crosshatching on a SiGe film grown on a $\mathrm{Si}(001)$ substrate studied by Raman mapping and atomic force microscopy," Phys. Rev. B 65, 233303 (2002).

${ }^{42}$ Note that as the difference in lattice parameter is measured against bulk Silicon and not bulk $S i_{x} G e_{1-x}$, we use the term deformation rather than strain to label quantified values, since part of the lattice difference is due to the composition rather than a mechanical stress.

${ }^{43} \mathrm{R}$. Berthelon, Strain integration and performance optimization in sub-20nm FDSOI CMOS technology, PhD, Université Paul Sabatier - Toulouse III (2018), issue: 2018TOU30066.

${ }^{44}$ The theoretical (113) deformation of $S i_{0.74} G e_{0.24}$ relative to bulk Silicon is $1.31 \%$ for a biaxal stress and $1.115 \%$ for an uniaxal one. 
(a) $\Delta(2 \vartheta)$

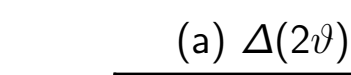

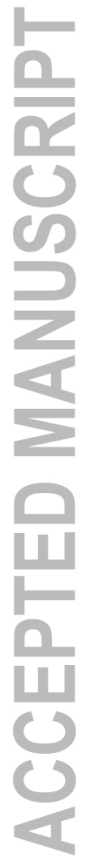

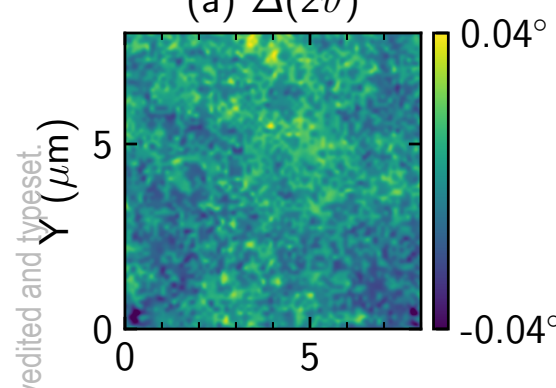

(b) Azimuthal tilt

(c) Planar tilt

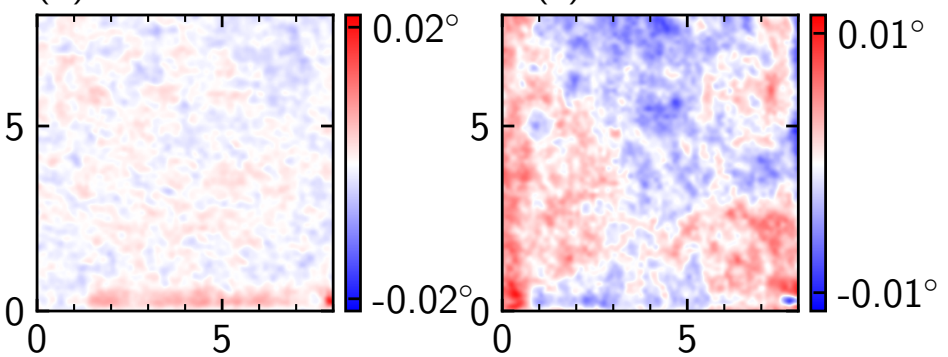

(a') $\Delta(2 \vartheta)$

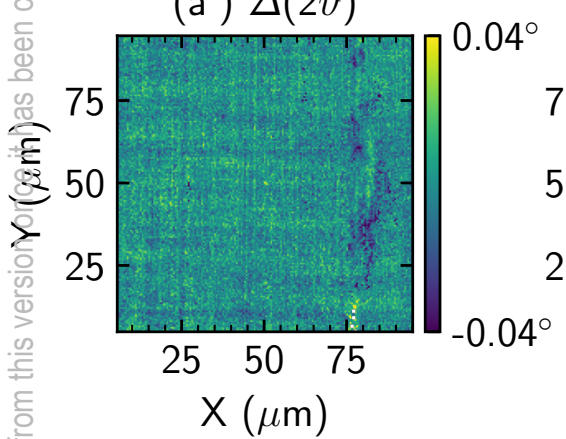

(b') Azimuthal tilt

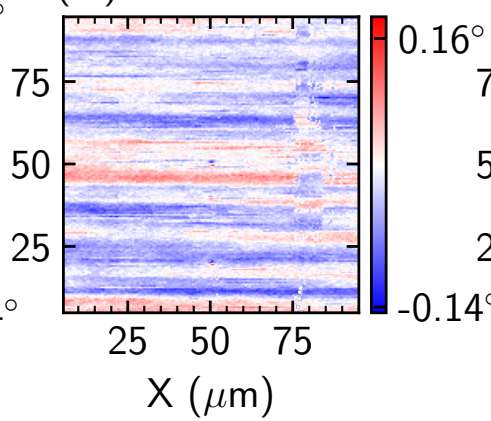

(c') Planar tilt

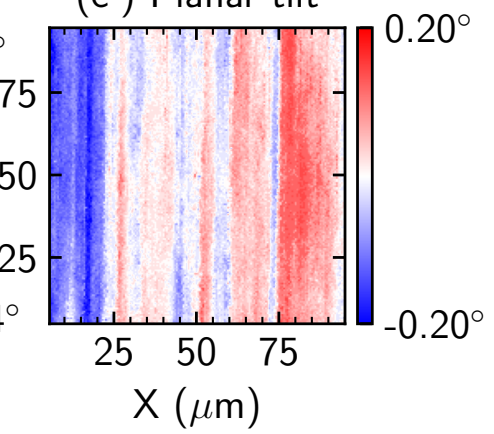


(a) I Obs

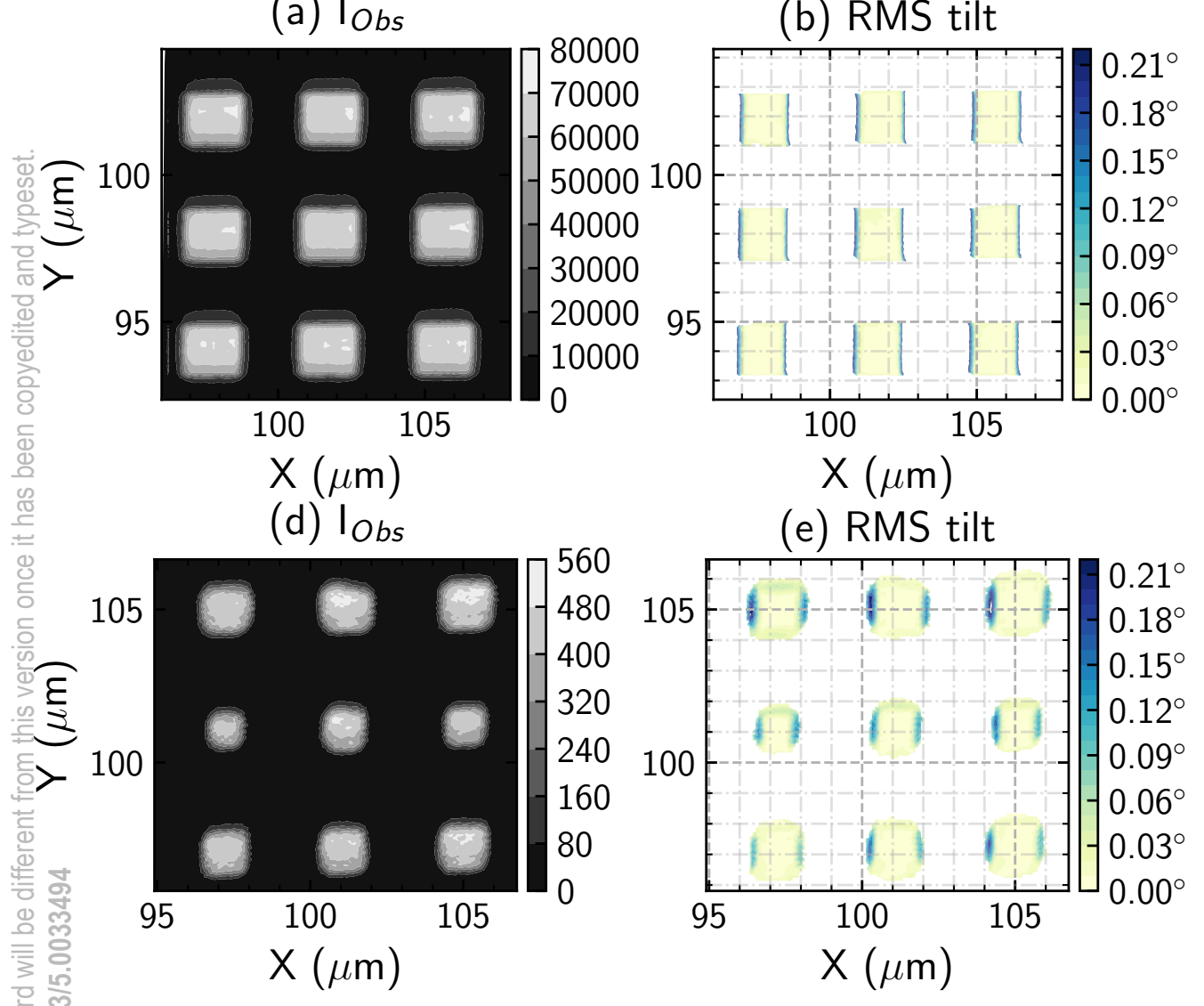

(c) $\epsilon_{113}=\left(a-a_{S i}\right) / a_{S i}$

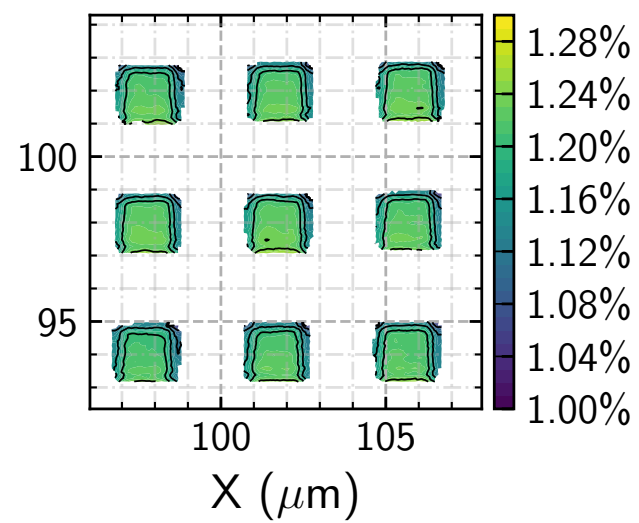

(f) $\epsilon_{004}=\left(a-a_{s i}\right) / a_{s i}$

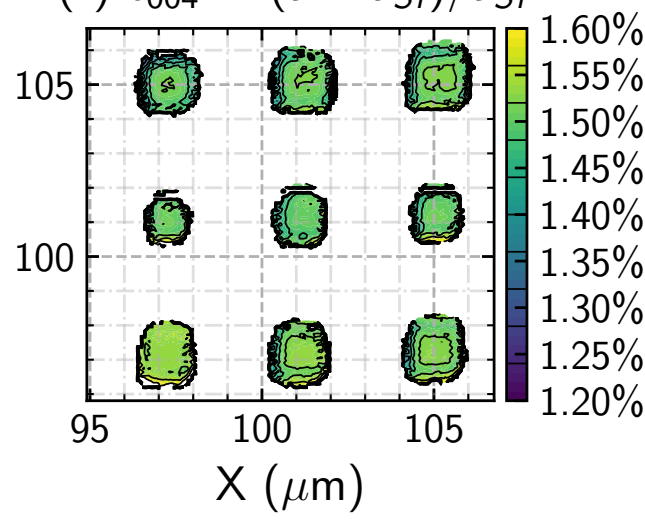


$\mathrm{I}_{\text {Obs }}$

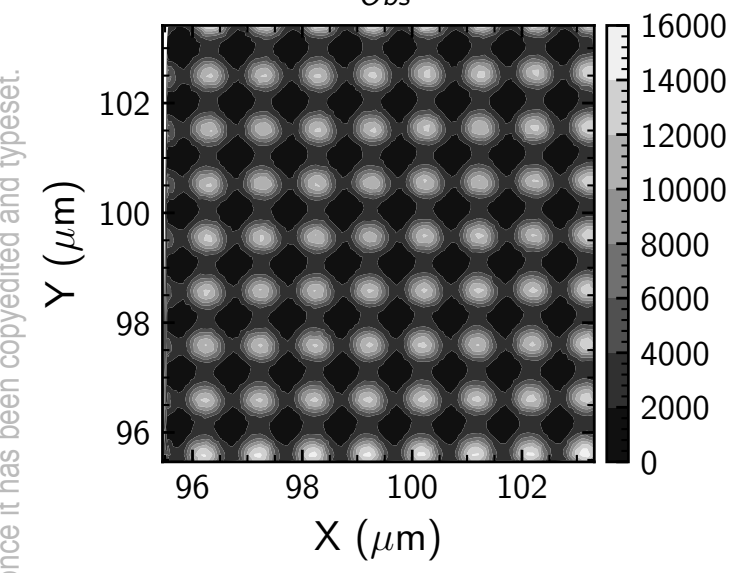

RMS tilt

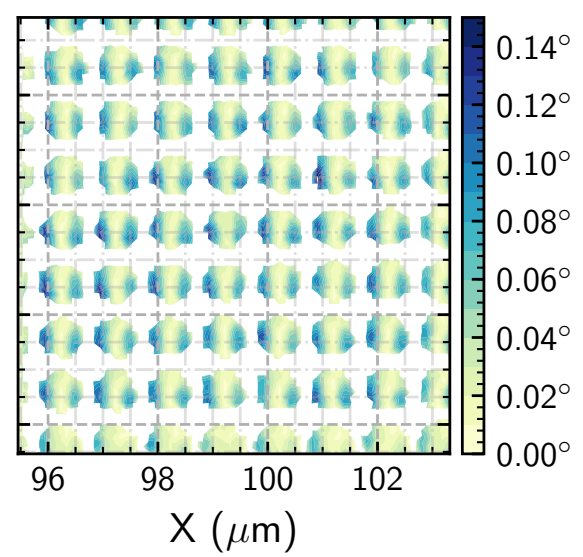

$\epsilon_{113}=\left(a-a_{S i}\right) / a_{S i}$

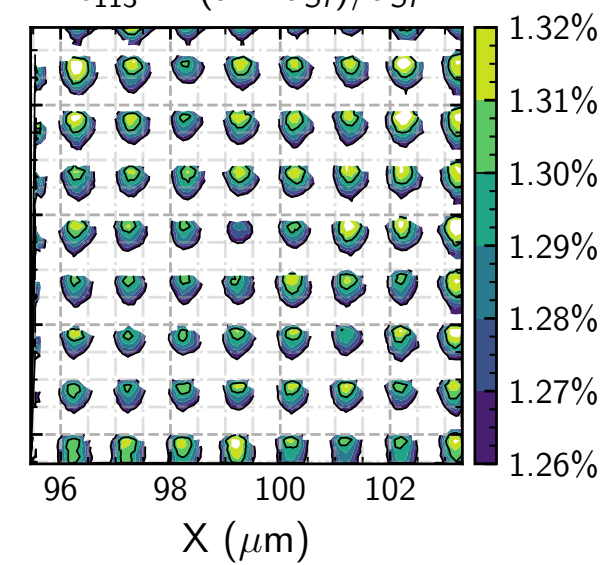



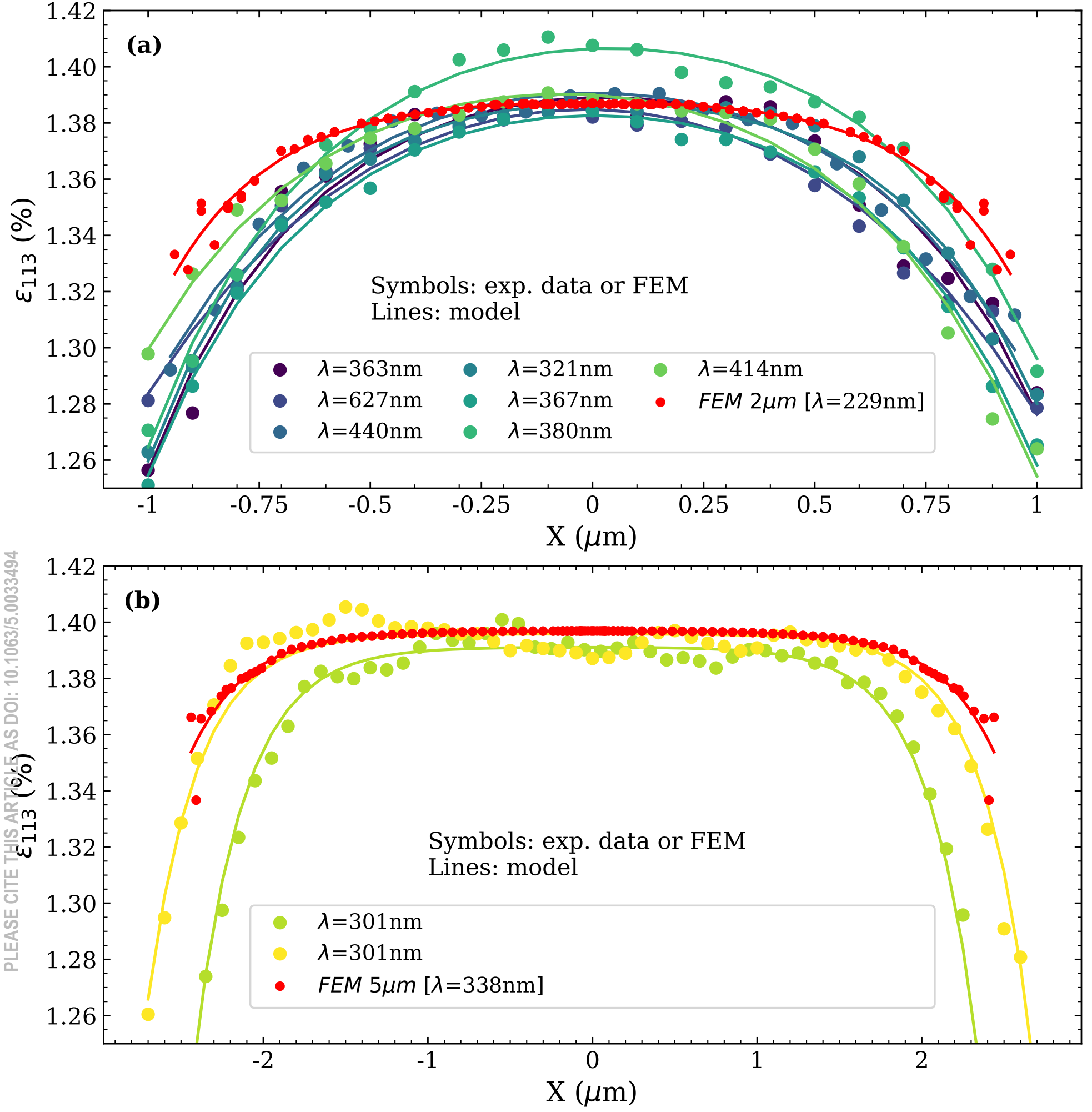